\title{
Epidemiology and evolution of Middle East respiratory syndrome coronavirus, 2012-2020
}

\author{
An-Ran Zhang 1,2,3, Wen-Qiang Shi ${ }^{2}$, Kun Liư ${ }^{4}$, Xin-Lou Li ${ }^{5}$, Ming-Jin Liü ${ }^{3}$, Wen-Hui Zhang ${ }^{2}$, Guo-Ping Zhao ${ }^{6}$, \\ Jin-Jin Chen ${ }^{2}$, Xiao-Ai Zhang ${ }^{2}$, Dong Miao ${ }^{2}$, Wei Ma ${ }^{1^{*}}$, Wei Liu ${ }^{2^{*}}$, Yang Yang ${ }^{3^{*}}$ and Li-Qun Fang ${ }^{2^{*}}$ (D)
}

\begin{abstract}
Background: The ongoing transmission of the Middle East respiratory syndrome coronavirus (MERS-CoV) in the Middle East and its expansion to other regions are raising concerns of a potential pandemic. An in-depth analysis about both population and molecular epidemiology of this pathogen is needed.

Methods: MERS cases reported globally as of June 2020 were collected mainly from World Health Organization official reports, supplemented by other reliable sources. Determinants for case fatality and spatial diffusion of MERS were assessed with Logistic regressions and Cox proportional hazard models, respectively. Phylogenetic and phylogeographic analyses were performed to examine the evolution and migration history of MERS-CoV.
\end{abstract}

Results: A total of 2562 confirmed MERS cases with 150 case clusters were reported with a case fatality rate of $32.7 \%$ (95\% Cl: 30.9-34.6\%). Saudi Arabia accounted for $83.6 \%$ of the cases. Age of $\geq 65$ years old, underlying conditions and $\geq 5$ days delay in diagnosis were independent risk factors for death. However, a history of animal contact was associated with a higher risk (adjusted $O R=2.97,95 \%$ Cl: 1.10-7.98) among female cases $<65$ years but with a lower risk (adjusted $O R=0.31,95 \% \mathrm{Cl}: 0.18-0.51$ ) among male cases $\geq 65$ years old. Diffusion of the disease was fastest from its origin in Saudi Arabia to the east, and was primarily driven by the transportation network. The most recent subclade C5.1 (since 2013) was associated with non-synonymous mutations and a higher mortality rate. Phylogeographic analyses pointed to Riyadh of Saudi Arabia and Abu Dhabi of the United Arab Emirates as the hubs for both local and international spread of MERS-CoV.

Conclusions: MERS-CoV remains primarily locally transmitted in the Middle East, with opportunistic exportation to other continents and a potential of causing transmission clusters of human cases. Animal contact is associated with a higher risk of death, but the association differs by age and sex. Transportation network is the leading driver for the spatial diffusion of the disease. These findings how this pathogen spread are helpful for targeting public health surveillance and interventions to control endemics and to prevent a potential pandemic.

\footnotetext{
*Correspondence: weima@sdu.edu.cn; Iwbime@163.com; yangyang@ufl.edu;

fang_lq@163.com

1 Department of Epidemiology, School of Public Health, Cheeloo College

of Medicine, Shandong University, 44 West Wenhua Road, Jinan, People's

Republic of China

${ }^{2}$ State Key Laboratory of Pathogen and Biosecurity, Beijing Institute

of Microbiology and Epidemiology, 20 Dong-Da Street, Fengtai District,

Beijing 100071, People's Republic of China

${ }^{3}$ Department of Biostatistics, College of Public Health and Health

Professions, and Emerging Pathogens Institute, University of Florida,

Gainesville, FL, USA

Full list of author information is available at the end of the article
}

(c) The Author(s) 2021, corrected publication 2021. Open Access This article is licensed under a Creative Commons Attribution 4.0 International License, which permits use, sharing, adaptation, distribution and reproduction in any medium or format, as long as you give appropriate credit to the original author(s) and the source, provide a link to the Creative Commons licence, and indicate if changes were made. The images or other third party material in this article are included in the article's Creative Commons licence, unless indicated otherwise in a credit line to the material. If material is not included in the article's Creative Commons licence and your intended use is not permitted by statutory regulation or exceeds the permitted use, you will need to obtain permission directly from the copyright holder. To view a copy of this licence, visit http://creativecommons.org/licenses/by/4.0/. The Creative Commons Public Domain Dedication waiver (http://creativecommons.org/publicdomain/zero/1.0/) applies to the data made available in this article, unless otherwise stated in a credit line to the data. 
Keywords: Middle East respiratory syndrome, MERS-CoV, Case fatality rate, Spatial diffusion, Phylogeny,

Phylogeographic dynamic

\section{Background}

Middle East respiratory syndrome (MERS) is a respiratory infectious disease first discovered in the Kingdom of Saudi Arabia in September 2012 [1]. The disease is caused by the Middle East respiratory syndrome coronavirus (MERS-CoV) which can be highly pathogenic in humans. Individuals infected with MERS-CoV may experience none, mild or severe respiratory illnesses or even death. As of 30 May 2020, a total of 27 countries in the Middle East, North Africa, Europe, Northeast Asia, and North America have reported 2562 laboratory-confirmed MERS cases and 881 associated deaths, according to the World Health Organization (WHO) [2]. The vast majority of MERS cases were reported by the Saudi Arabia, followed by Republic of Korea [2]. Frequent travelers and worshippers from and to the Middle East have raised the concern about a global pandemic, given the lack of effective treatment and vaccine [3]. In February 2018, WHO formally incorporated MERS into the Research and Development Blueprint (the R\&D Blueprint) to promote research in this area [4].

Current epidemiological studies suggest that humanto-human transmission of MERS-CoV is inefficient, and the primary infection mode is via direct/indirect contact with dromedary camels, although other mammals may also serve as the reservoir [5-10]. On the other hand, human clusters of MERS have been continuously observed in healthcare and household settings, especially among people with chronic conditions or compromised immunity $[11,12]$. Several studies explored risk factors for the transmission of MERS-CoV at the individual level in specific countries and found that infection risk was mainly driven by recent exposure to dromedary or its raw products, chronic conditions, or close contact with other MERS patients [11, 13-16]. However, very few studies have systematically analyzed spatial diffusion of the virus at the population level and associated risk factors. In addition, while it has been shown that chronic condition and male sex are highly predictive of fatal outcomes [17], no study has examined potential interactions among key predictors for death, e.g., demographic characteristics and animal contact. Some of these predictors are correlated, e.g., males tend to have much more frequent contact with dromedary. Consequently, it is necessary to condition on one predictor when evaluating the effect of another.

In the midst of the pandemic of severe acute respiratory syndrome coronavirus 2 (SARS-CoV-2), it is crucial to understand the epidemiological characteristics and evolutionary history for MERS-CoV as the two coronaviruses are genetically related. The possibility of recombination between the two viruses if co-infection of the same host occurs cannot be totally ruled out, as their host species do overlap, e.g., humans and bats [18]. Thus far, phylogenetic and phylogeographic analyses focusing on MERS-CoV have been either outdated or restricted to small data sets $[9,10,19-21]$. In addition, some evolutionary characteristics of MERS-CoV such as which genes are subject to positive selection, need to be closely monitored.

By assembling MERS surveillance and contact tracing data up to June of 2020 from public health agencies and peer-reviewed literature, we summarized the epidemiological features and spatiotemporal spread of MERS around the globe. We investigated risk factors for fatality and how their effects could be modified by each other. In addition, we assessed the roles of a variety of environmental, socioeconomic, and biological factors in the spatial diffusion of MERS-CoV. Using publicly available MERS-CoV full-genome sequences, we further assessed the evolution and migration history of the virus. Piecing these results together, we aim to provide an up-to-date picture about both population and molecular epidemiology of this pathogen.

\section{Method}

\section{Data collection and management}

We assembled three datasets: (1) a list of individual human cases worldwide with demographic, exposure and clinical information, (2) eco-geographic and socioeconomic characteristics (referred to as socioenvironmental variables hereinafter) at the appropriate administrative level in the Middle East (county for Saudi Arabia and province for the other countries), and (3) full-genome sequences of MERS-CoV worldwide.

Data on confirmed MERS cases were collected from official reports of WHO, the Food and Agriculture Organization (FAO) of the United Nations, and the health departments of affected countries, which were cross-validated with and supplemented by data from websites and literatures. Search medical subject headings $(\mathrm{MeSH})$ terms used were "Middle East Respiratory Syndrome Coronavirus/MERS-CoV/HCoV-EMC" or "Middle East respiratory syndrome/Middle Eastern Respiratory Syndrome/MERS". 
All the cases had been confirmed following a standard WHO technical guidance (https://www.who.int/csr/ disease/coronavirus_infections/case_definition/en/). A valid record of MERS case must include the basic demographic information (gender, age, reporting country, city of residence, being healthcare worker or not, baseline chronic conditions), dates of critical events (such as symptom onset, first hospitalization, laboratory confirmation), and exposure information (whether exposed to animal or its raw production, or exposed to confirmed MERS patients). Cases without any individual information, duplicated records and no-confirmed cases were removed. Each confirmed case was geo-referenced and mapped according to the finest address available using GIS technologies. Thematic maps of cumulative numbers of confirmed MERS cases and clusters were created using ArcGIS 10.5 (Esri Inc, Redlands, CA, USA).

The following socioenvironmental variables potentially related to the transmission of MERS were collected: population density, camel density, monthly meteorological data, elevation, land cover, economic development level, transportation, locations of hospitals. Full-genome sequences (>30 $000 \mathrm{bp}$ ) of MERS-CoV up to June of 2020, together with isolation year, host type and location, were retrieved from GenBank. Protein and the coding sequences (CDS) sequences of these MERS-CoV sequences were derived. All the data sources (Additional file 1: Table S1) involved in this study were introduced in detail in the Additional file 1: Additional Appendix.

\section{Descriptive analysis}

MERS-affected countries were grouped into four categories of transmission type: (i) zoonotic transmission plus human-to-human transmission, (ii) zoonotic transmission without human-to-human transmission, (iii) imported infection plus human-to-human transmission, and (iv) imported infection without human-to-human transmission (Additional file 1: Table S2). Existence of zoonotic transmission was determined by reported endemic circulation with frequent zoonotic introduction. A case cluster is defined as a group of two or more epidemiologically linked cases, where epidemiological link refers to close contact as indicated by the source database or literature. For example, the WHO database provides the ID number of the source case for some cases. By this definition, all cases in the outbreak in Republic of Korea can be traced to the same imported case and are therefore considered a single case cluster [13]. Demographic characteristics were compared between country categories and between case types using the Kruskal-Wallis test for continuous variables and the $\chi^{2}$ test for categorical variables.

\section{Analysis of risk factors for cases fatality}

Logistic regression was performed to explore risk factors associated with the survival outcome of MERS cases. All MERS cases with reliable survival outcome and contact information (before symptom onset) were included in the analysis. The following potential risk factors were included in the analysis: age group ( $\geq 65$ years old vs $<65$ years old), sex (male vs female), region (Middle East vs other), occupation (healthcare worker vs other), underlying chronic conditions including diabetes mellitus, renal failure, chronic respiratory and circulatory diseases and compromised immune systems (yes, no, unknown), animal contact (yes, no, unknown), time from disease onset to confirmation (OTC, $>5$ days vs $\leq 5$ days), and reporting year (2012-2014, 2015-2016, 2017-2020). Among variables with missing values, OTC were imputed using $\mathrm{R}$ package "mice". But missing values of underlying condition and animal contact were treated into a category of unknown, for imputation efficiency is not high for variables with a missing rate over $30 \%$, Backward elimination was used to a parsimonious model (threshold $P$-value $=0.05)$. Two-way interactions among age group, sex and animal contact were considered based on the amount of data per category of each factor.

\section{Spatiotemporal diffusion}

We limited the spatiotemporal diffusion analysis to the Middle East Region, the main endemic region of MERS. Opportunistic long-distance exportations of MERS cases, e.g., to Europe and Republic of Korea, were not considered. The spatial unit used in this study is the second-level administrative unit, e.g., province, for most countries. Provinces of Saudi Arabia are much larger than those of neighboring countries. To make spatial units comparable between countries, we use the third-level administrative area (county) as the spatial unit for Saudi Arabia. Finally, a total of 283 administrative units were included in the spatiotemporal diffusion analysis.

We obtained 34 socioenvironmental variables from various data sources (Additional file 1: Table S3). Camel density was available only at the national level in many countries. Considering the role of camel as a MERS-CoV reservoir, we imputed missing values of camel density using the $\mathrm{R}$ package "mice". To account for uncertainties in these missing values, we generated 100 imputed sets of camel density and averaged all analyses related to camel density over these imputation sets. To reduce collinearity among these variables, we screened pairwise correlations. If two variables have an absolute Pearson correlation higher than 0.65 , the one with a higher average correlation with all other variables was excluded. 
A Cox proportional hazard model was used to assess which socioenvironmental variables were associated with the reporting time (since September 2012) of the first MERS case in each space unit. A spatial trend contour plot was developed to visualize the spatial diffusion of the disease. The time between adjacent contours was fixed at 200 days, and a wider gap between adjacent contours indicates a faster spatial diffusion. All descriptive and diffusion analyses were performed using the $\mathrm{R}$ software version 3.6.2 ( $\mathrm{R}$ Foundation for Statistical Computing, Vienna, Austria). A two-sided $P$-value $<0.05$ was considered statistically significant.

\section{Phylogenetic and phylogeographic analyses}

The whole-genome sequences were analyzed using toolkits provided by the Nextstrain framework [22]. Sequences were aligned using MAFFT v7.407 [23], and the alignment was trimmed to a reference genome (GenBank accession ID: NC_019843.3). A phylogenetic tree was built using a maximum likelihood approach implemented in IQ-Tree v1.6.10 [24]. For the phylogeographic analysis, TreeTime was used to infer the divergence time, discrete traits of the ancestral nodes (location and host), and geographic transmission history across the tree [25]. To detect sites under positive selection among CDS of protein genes, for each gene, the original open reading frame (ORF) sequences were first aligned using MAFFT and CDS were aligned using PAL2NAL v14 under the guidance for protein alignment [26]. CodeML in PAML $\mathrm{v} 4.9$ as part of the ETE 3 package ( $\mathrm{v}$ 3.1.1) was used to detect positive selection sites by branch-site test $[27,28]$. To balance the sample size of each host species, human and camel sequences were randomly down-sampled to five sequences per host species. After smoothing mortality rate and incidence rate over space and time, we matched these smoothed rates with tree tips (MERS-CoV sequences) by specimen collection year and location to assess potential association of phylogeny with mortality and incidence rates.

\section{Results}

\section{Epidemiological features of MERS cases}

From September 2012 to June 2020, a total of 2562 laboratory-confirmed MERS cases were reported. After excluding 112 cases with incomplete data, 2450 confirmed MERS cases, together with 150 derived case clusters, were included in subsequent analyses (Table 1). The median age was 53 years old (IQR: 38-65), and 69.4\% of cases were male. Healthcare workers accounted for $13.7 \%(335 / 2450)$ of the total patients. The median time from disease onset to diagnosis was 5 days (IQR: 3-8). Death occurred in 802 patients, leading to a case fatality rate (CRF) of $32.7 \%$ (95\% CI: 30.9-34.6\%). Among the
1453 patients with known exposure history, 356 (24.0\%) reported animal contact. Zoonotic infections (i.e., countries in transmission categories i and ii) only occurred in the Middle East, although cases with animal contact had also been imported into Europe and Southeast Asia (Fig. 1). Among countries with locally infected patients, the proportion of cases with animal contact exceeded $50 \%$ in Qatar, followed by 15-29\% in Oman, United Arab Emirates (UAE), and Saudi Arabia (Fig. 1).

Saudi Arabia, Republic of Korea and UAE together reported the vast majority of the cases and case clusters (Additional file 1: Table S2 and Figure S1-S2). Patients from the three countries shared similar age and sex distributions, but Saudi Arabia had the highest CFR (35.1\%), probably due to a higher proportion of underlying conditions (Table 1). Republic of Korea had the highest proportion (>99\%) of hospital infections and the shortest time (median $=4$ days) from disease onset to diagnosis, yet its CFR (18.8\%) is comparable to that of UAE (16.1\%). UAE had the highest proportion of asymptomatic infections and the longest survival time among fatal cases (Table 1).

Cases with animal contact were older, more likely to be male, and more likely to have underlying conditions and a longer delay from disease onset to diagnosis, in comparison to cases without (Additional file 1: Table S4). These characteristics of cases with animal contact could partially explain their significantly higher CFR (35.1\% vs $24.3 \%, P<0.001)$ than those without animal contact (Additional file 1: Table S4). However, among fatal cases, those with animal contact had a slightly longer survival time than those without (median $=11.5$ vs 9 days, $P<0.001)$. Cases with only patient contact were more likely to be health-care workers and more likely to be asymptomatic (Additional file 1: Table S4). The proportion of cases with animal contact was increasing from 2012 to 2018 and seemed to stabilize thereafter. Epidemics peaked mostly between April and September (Additional file 1: Figure S3). In contrast, cases with animal contact were more likely to occur from January to March. Seasonality differed slightly between countries, e.g., peaks of zoonotic infections in UAE were also observed in May. The peak of the global epidemic in June of 2015 was due to the opportunistic outbreak in Republic of Korea, which does not necessarily reflect seasonality in the endemic setting.

\section{Determinants for case fatality}

All the eight individual-level factors (age group, sex, living in the Middle East, healthcare worker, underlying conditions, animal contact history before onset, delay from disease onset to diagnosis and onset year) were significantly associated with mortality in the univariate analysis, with age and underlying condition as 
Table 1 Baseline demographic and clinical characteristics of confirmed Middle East respiratory syndrome cases reported between September 2012 and June 2020

\begin{tabular}{|c|c|c|c|c|c|}
\hline & \multicolumn{5}{|c|}{ Number (\%) of cases } \\
\hline & Total & Saudi Arabia & Korea & United Arab Emirates & Others \\
\hline Number of confirmed cases & 2450 & 2048 & 186 & 93 & 123 \\
\hline Annual incidence $\left(/ 10^{7}\right)$ & 0.45 & 94.34 & 4.71 & 14.07 & 0.023 \\
\hline Female & $751(30.7)$ & $626(30.6)$ & $75(40.3)$ & $20(21.5)$ & $30(24.4)$ \\
\hline Age, years (median, IQR) & $53(38-65)$ & $53(38-65)$ & $55(42-66)$ & $43(33-59)$ & $52(38-65)$ \\
\hline Number of case cluster & 150 & 123 & 1 & 11 & 15 \\
\hline Number of deaths (CFR, \%) & $802(32.7)$ & $718(35.1)$ & $35(18.8)$ & $15(16.1)$ & $34(27.6)$ \\
\hline \multicolumn{6}{|l|}{ Contact history } \\
\hline Animals & $356(14.5)$ & $310(15.1)$ & 0 & $16(17.2)$ & $30(24.4)$ \\
\hline Patients & $1097(44.8)$ & $820(40.1)$ & $184(98.9)$ & $47(50.5)$ & $46(37.4)$ \\
\hline Unknown & $997(40.7)$ & $918(44.8)$ & $2(1.1)$ & $30(32.3)$ & $47(38.2)$ \\
\hline \multicolumn{6}{|l|}{ Occupation } \\
\hline Healthcare worker & $335(13.7)$ & $265(12.9)$ & $30(16.1)$ & $26(28.0)$ & $14(11.4)$ \\
\hline Others & $2115(86.3)$ & $1783(87.1)$ & $156(83.9)$ & $67(72.0)$ & $109(88.6)$ \\
\hline \multicolumn{6}{|l|}{ Exposure site } \\
\hline Hospital & $724(29.6)$ & $496(24.2)$ & $185(99.5)$ & $21(22.6)$ & $22(17.9)$ \\
\hline Household & $120(4.9)$ & $93(4.5)$ & 0 & $11(11.8)$ & $16(13.0)$ \\
\hline Others & $1606(65.5)$ & $1459(71.3)$ & $1(0.5)$ & $61(65.6)$ & $85(69.1)$ \\
\hline Asymptomatic infection & $201(8.2)$ & $173(8.5)$ & 0 & $19(20.4)$ & $9(7.3)$ \\
\hline Underlying condition & $1272(51.9)$ & $1156(56.5)$ & $20(10.8)$ & $38(40.9)$ & $58(47.2)$ \\
\hline $\begin{array}{l}\text { Time from disease onset to diagno- } \\
\text { sis, days (median, IQR) }\end{array}$ & $5(3-8)$ & $5(3-8)$ & $4(2-8)$ & $8(6-14)$ & $8(5-13.5)$ \\
\hline $\begin{array}{l}\text { Time from disease onset to death, } \\
\text { days (median, IQR) }\end{array}$ & $11(7-17)$ & $10(6-16)$ & $12(9-16)$ & $21.5(18-27)$ & $16(11-20)$ \\
\hline \multicolumn{6}{|l|}{ Year of occurrence } \\
\hline 2012 & $9(0.4)$ & $5(0.2)$ & 0 & 0 & $4(3.3)$ \\
\hline 2013 & $169(6.9)$ & $137(6.7)$ & 0 & $12(12.9)$ & $20(16.3)$ \\
\hline 2014 & $652(26.6)$ & $560(27.3)$ & 0 & $59(63.3)$ & $33(26.8)$ \\
\hline 2015 & $680(27.8)$ & $457(22.3)$ & $185(99.5)$ & $7(7.5)$ & $31(25.2)$ \\
\hline 2016 & $254(10.4)$ & $242(11.8)$ & 0 & $3(3.2)$ & $9(7.3)$ \\
\hline 2017 & $250(10.2)$ & $237(11.6)$ & 0 & $7(7.5)$ & $6(4.9)$ \\
\hline 2018 & $150(6.1)$ & $145(7.1)$ & $1(0.5)$ & $1(1.2)$ & $3(2.4)$ \\
\hline 2019 & $226(9.2)$ & $208(10.2)$ & 0 & $2(2.2)$ & $16(13.0)$ \\
\hline 2020 & $60(2.4)$ & $57(2.8)$ & 0 & $2(2.2)$ & $1(0.8)$ \\
\hline
\end{tabular}

$\mathrm{IQR}$, interquartile range; $\mathrm{CFR}$, case fatality rate

the leading factors with $O R>5$ (Table 2). We identified a multivariate model containing all eight main effects, as well as two-way interactions among age group, sex and animal contact. After controlling for other factors, underlying condition was associated with 3.5fold increase in the risk $(O R=3.5,95 \% C I: 2.5-4.89)$, whereas being a healthcare worker was protective $(O R=0.33,95 \%$ CI: 0.21-0.54). OTC $\geq 5$ days increased the risk by $31 \%$ (95\% CI: 5-63\%). Compared to $2012-$ 2014, the risk of death was higher during 2015-2016 $(O R=1.63$, 95\% CI: 1.27-2.09) but lower during 20172019 (OR $=0.70,95 \%$ CI: 0.53-0.92).
Odds ratios with regard to each of age group, sex and animal contact while controlling for the other two are shown in Additional file 1: Table S5-S7. The older age group ( $\geq 65$ years) was associated with a higher risk of death in general, with significant ORs ranging 1.64-10.52 for all combinations of sex and animal contact history (Additional file 1: Table S5). However, the age effect was more prominent among patients without animal contact. Among patients without animal contact, the adjusted $O R$ were 5.65 (95\% CI: 3.75-8.50) for males and 10.52 (95\% CI: 6.46-17.11) for females, compared to 1.64 (95\% CI: $1.03-2.62)$ for males and 3.06 


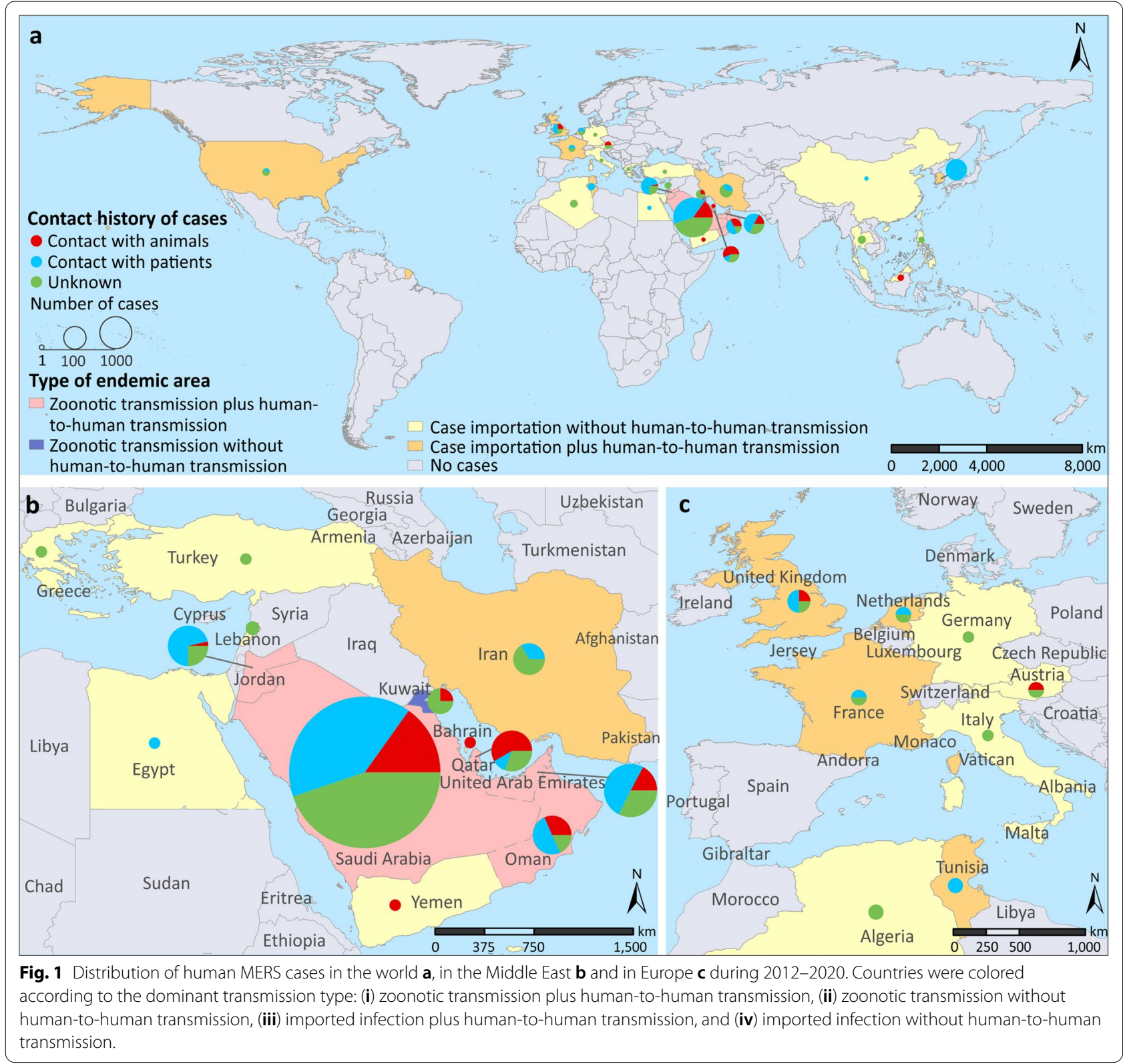

(95\% CI: 1.59-5.86) for females among those with animal contact. From these estimates, it is also clear that the age effect was more prominent among female patients compared to male patients. Sex did not affect the risk of death significantly, although males tended to have higher risk among the younger age group without animal contact (adjusted $O R=2.34,95 \%$ CI: $1.57-3.49$, Additional file 1: Table S6). A history of animal contact was associated with a higher risk (adjusted $O R=2.97,95 \%$ CI: $1.10-7.98)$ among female cases $<65$ years but with a lower risk (adjusted $O R=0.31,95 \% C I$ : $0.18-0.51$ ) among male cases $\geq 65$ years old (Additional file 1: Table S7). The model-estimated effects of age group, sex, and underlying conditions on CFR are in line with the observed CFRs stratified by each of these variables (Additional file 1: Figure S4). The CFR decreased gradually since 2015.

\section{Geographic expansion}

After the screening of correlations, 20 out of the 34 socioenvironmental variables (Additional file 1: Table S3) remained for further analysis: population density, elevation, camel density, 8 variables regarding land covers (cropland, forest, shrubland, grassland, wetland, bareland, waterbody, urban), 6 ecoclimatic variables (bio1, 
Table 2 Logistic regression analyses of potential risk factors for mortality among Middle East respiratory syndrome cases, September 2012-June 2020

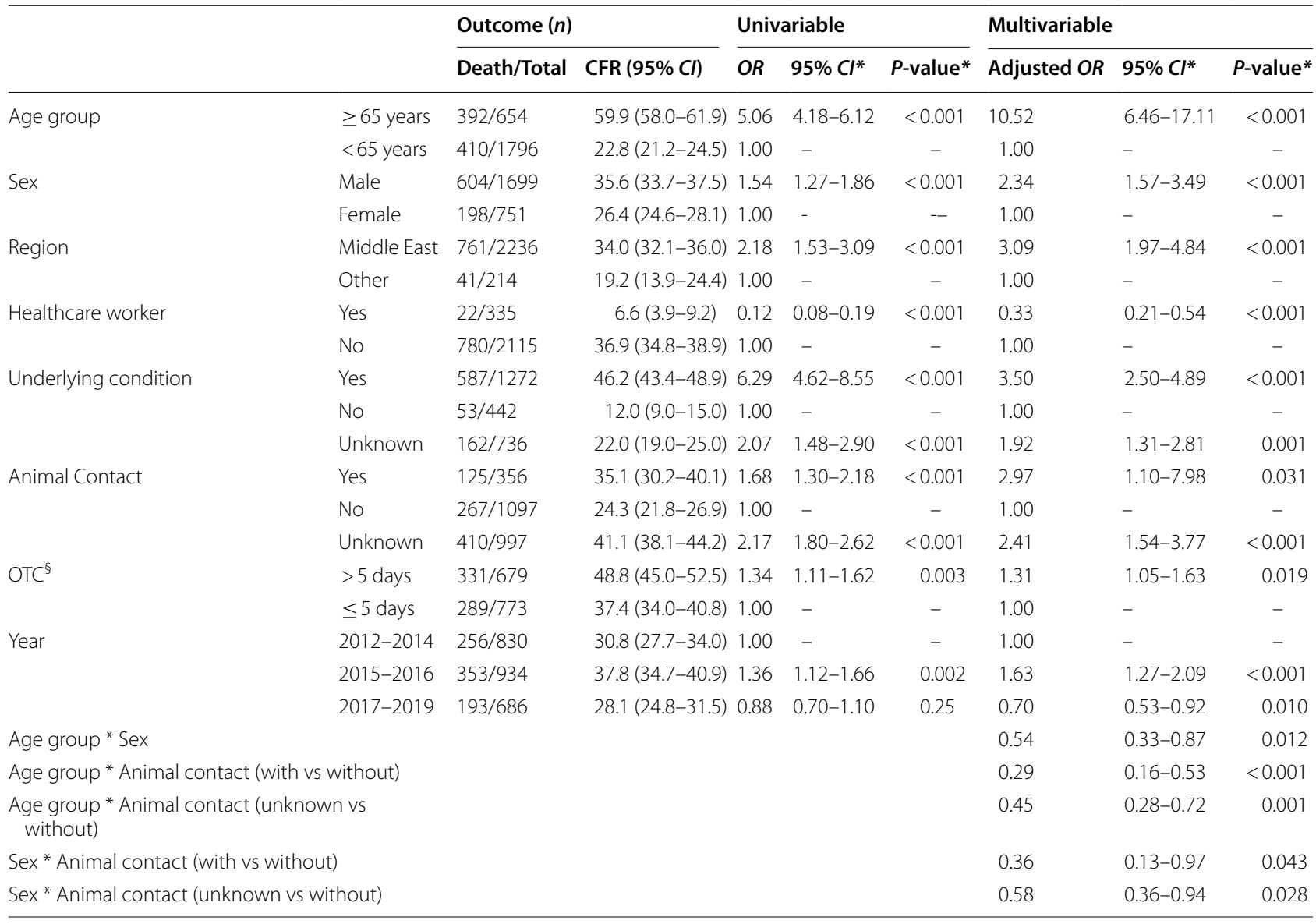

CFR case fatality rate, OR odds ratio, $\mathrm{Cl}$ confidence Interval, OTC Time from disease onset to confirmation

§OTC have missing values. Description (column 2-5) are based on non-missing values. But logistic analysis were based on data after interpolation

*Means that there must be no corresponding results for being the control groups

bio2, bio3, bio4, bio5, bio12), transportation (railway, main road) and number of hospitals.

The first case was reported in Bisha, central-west Saudi Arabia in September 2012. The disease spread more rapidly towards the east (UAE and Oman) than towards other directions (Fig. 2a). The diffusion appears to be acerating in recent years. At the second administrative level, 12 of the 14 eco-geographic variables were associated with the spread of MERS in the univariable Cox regressions (Table 3 ). In the multivariate analysis, positive associations with the disease diffusion were found for seven factors, and the top three drivers are intersection with main roads [adjusted hazard ratio $(\mathrm{HR})=15.45$, 95\% CI: 2.11-113.26, $P=0.007$ ], intersection with railways (adjusted $\mathrm{HR}=2.33$, 95\% CI:1.37-3.97, $P=0.002$ ) and elevation (adjusted $\mathrm{HR}=2.40,95 \% \mathrm{CI}: 1.47-3.91$, $P<0.001)$. A higher coverage of cropland seemed to have impeded the disease diffusion (adjusted $\mathrm{HR}=0.51,95 \%$
CI: $0.27-0.95, P=0.034)$. We overlaid the land coverage and transportation networks with the spatiotemporal distribution of the first reported cases in each space unit (Fig. 2b). In the Arabian Peninsula, intersection with major roads and railroads was clearly associated with earlier invasion.

\section{Phylogeny and phylogeographic analysis of whole-genome sequences}

In total, 499 MERS-CoV full-genome sequences were obtained from GenBank, including 251 sequences from human patients, 237 from camel, seven from bat, three from hedgehog, and one from Lama glama (llama). These sequences were collected between 2011 and 2019 from 15 countries, and $90.0 \%$ of them were from Middle East. IQ-Tree selected the GTR and FreeRate with ten categories as the best substitution model for these sequences. 


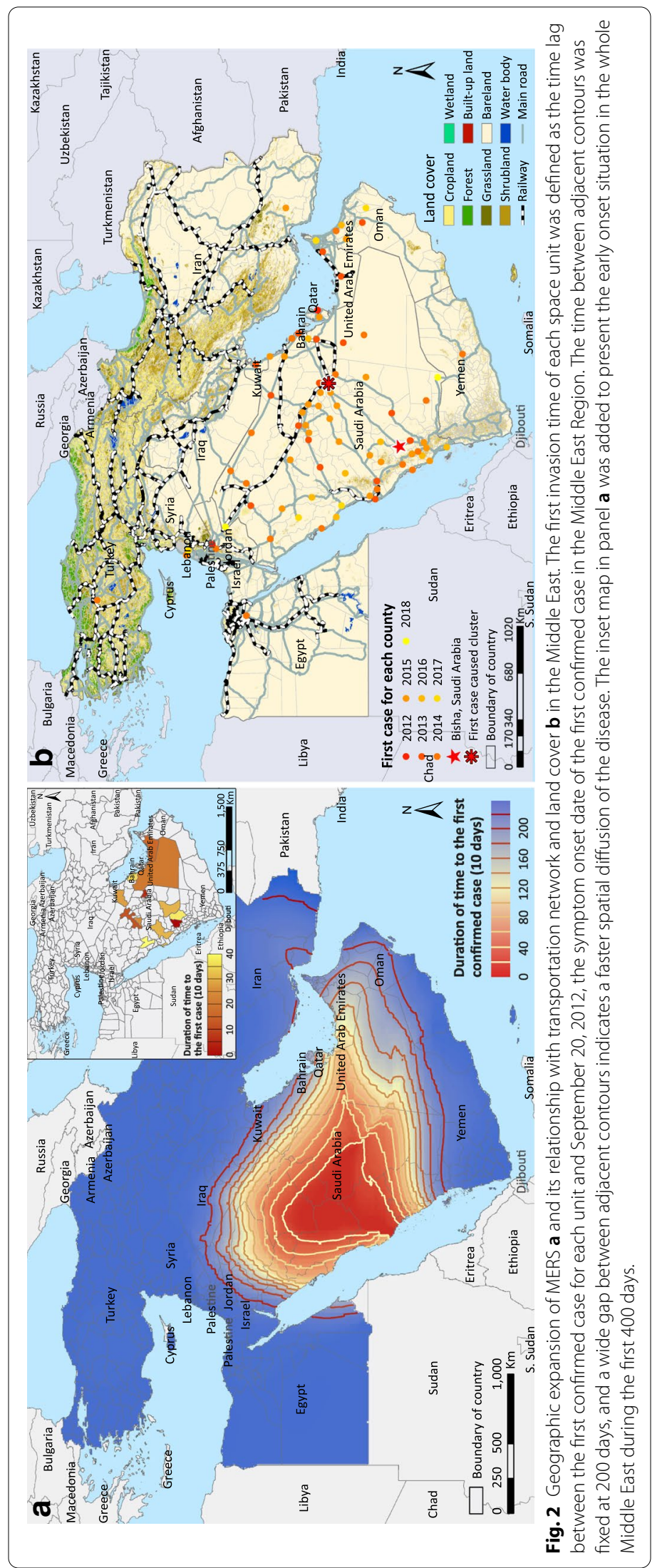


Table 3 Survival analyses on the time to the first reported Middle East respiratory syndrome case in each space unit, September 2012-June 2020

\begin{tabular}{|c|c|c|c|c|c|c|}
\hline \multirow[t]{2}{*}{ Predictors } & \multicolumn{3}{|l|}{ Univariable } & \multicolumn{3}{|l|}{ Multivariable } \\
\hline & Hazard ratio & $95 \% \mathrm{Cl}$ & $P$-value & Hazard ratio & $95 \% \mathrm{Cl}$ & $P$-value \\
\hline Population density $\left(10 / \mathrm{km}^{2}\right)$ & 1.00 & $0.99-1.00$ & 0.44 & & & \\
\hline Camel density $\left(10 / \mathrm{km}^{2}\right)$ & 1.74 & $1.35-2.23$ & $<0.001$ & & & \\
\hline Elevation (km) & 0.99 & $0.66-1.46$ & 0.94 & 2.40 & $1.47-3.91$ & $<0.001$ \\
\hline \multicolumn{7}{|l|}{ Meteorological variable } \\
\hline $\mathrm{BIO} 1$ & 1.58 & $1.48-1.68$ & $<0.001$ & 1.38 & $1.25-1.52$ & $<0.001$ \\
\hline $\mathrm{BlO} 2$ & 1.97 & $1.74-2.23$ & $<0.001$ & 1.28 & $1.12-1.46$ & $<0.001$ \\
\hline $\mathrm{BlO} 3$ & 1.04 & $1.01-1.07$ & 0.012 & & & \\
\hline $\mathrm{BlO} 4$ & 1.003 & $1.002-1.005$ & $<0.001$ & & & \\
\hline $\mathrm{BlO5}$ & 1.36 & $1.30-1.43$ & $<0.001$ & & & \\
\hline $\mathrm{BIO} 12$ & 0.78 & $0.74-0.83$ & $<0.001$ & & & \\
\hline \multicolumn{7}{|c|}{ Percentages of land cover (10\%) } \\
\hline Cropland & 0.27 & $0.14-0.49$ & $<0.001$ & 0.51 & $0.27-0.95$ & 0.034 \\
\hline Shrubland & 0.20 & $0.07-0.58$ & 0.003 & & & \\
\hline Bare land & 1.49 & $1.39-1.59$ & $<0.001$ & 1.13 & $1.03-1.25$ & 0.014 \\
\hline Intersect with railway & 3.76 & $2.35-6.01$ & $<0.001$ & 2.33 & $1.37-3.97$ & 0.002 \\
\hline Intersect with main road & 59.53 & $8.27-428.25$ & $<0.001$ & 15.45 & $2.11-113.26$ & 0.007 \\
\hline Number of hospitals (10) & 1.28 & $1.15-1.37$ & $<0.001$ & 1.19 & $1.04-1.44$ & 0.010 \\
\hline
\end{tabular}

$\mathrm{BIO} 1$ annual average temperature, $\mathrm{BIO} 2$ average diurnal range of temperature, $\mathrm{BIO} 3$ isothermality, $\mathrm{BIO} 4$ temperature seasonality, $\mathrm{BIO} 5$ max temperature of warmest month, BIO12 Annual precipitation

Turkey and Iran are excluded in this analysis. Space unit is the third-level administrative area (county) in Saudi Arabia and the second-level administrative area elsewhere

\section{Phylogeny}

An initial analysis showed that sequences from bat and hedgehog form a separate clade distant from the main clade of sequences from humans, camels and Lama glama (Additional file 1: Figure S5a), confirming that camel is the major zoonotic reservoir of MERS-CoV for spillover to human. We excluded the ten sequences from bat and hedgehog from subsequent analyses. The main clade of human, camel and llama strains, named clade $\mathrm{C}$, contains five subclades numbered $\mathrm{C} 1-\mathrm{C} 5$, with $\mathrm{C} 5$ being the largest subclade with 398 sequences (Fig. 3, Additional file 1: Figure S5b). Overall, sequences from human and camel mixed throughout the whole tree, indicating multiple introduction events from camel to human. Nevertheless, the human and camel sequences sampled after 2016 in our database were genetically distant from each other. The root ancestor of clade C, dated back to January 2007 (confidence interval: April 2006-September 2008), was $49.3 \%$ likely from camel and $50.7 \%$ likely from human. The case mortality rates differed between clades in the phylogenetic tree. C5 was associated with a higher mortality rate than other clades (a difference of $1 \%$ in CFR, $P$-value $=2.0 \times 10^{-4}$, Fig. 3). A sub-clade of C5, C5.1, showed an even higher mortality rate than clades $\mathrm{C} 1-\mathrm{C} 4$ (a difference of
$4 \%$ in CFR, $P$-value $\left.<2.2 \times 10^{-16}\right)$. Compared to subclades $\mathrm{C} 1-\mathrm{C} 4$, we found non-synonymous mutations in regions encoding the ORF3 protein (P86L) and the NS4B protein (M6T) among the C5.1 sequences as well as in regions encoding the $1 \mathrm{AB}$ protein $(\mathrm{S} 6737 \mathrm{~N})$, the NS4A protein (P106S), and the Membrane protein (V69I) among other C5 sequences.

\section{Phylogeographic dynamics}

The spatiotemporal transmission pattern of clade $\mathrm{C}$ was characterized by intense local migration within the Middle East and occasional long distance exportation (Movie 1; Additional file 1: Figure S6). The top three most likely locations of the inferred root ancestor were Riyadh of Saudi Arabia, the Nile Delta region and Jordan with posterior probabilities of $31 \%, 17 \%$ and $12 \%$, respectively. Riyadh appeared to be the major source exporting infections both locally and internationally. It was estimated with $99 \%$ posterior probability as the location of common ancestral node for subclades C3, C4, and C5 which cover $97.5 \%$ of the collected sequences. Early exportation of the virus to Egypt and Jordan likely occurred before 2010. The circulation of MERS-CoV among dromedary camels in East Africa possibly started before 2010. The model inferred migration of the virus from Egypt to Ethiopia 
during 2011-2013 and subsequently to Kenya during 2014-2017, partly supported by a serological study conducted in Egypt in 2013 that found both domestic dromedary camels and those imported from Ethiopia were seropositive [29]. Intense migration of the virus from Riyadh towards local cities in Saudi Arabia, Abu Dhabi in UAE and Europe started during 2011-2012. Abu Dhabi soon joined Riyadh as the second hub exporting the virus to other Middle East cities as well as to Europe. The opportunistic exportation events from the Middle East to the United States in 2014 and to East Asia in 2015 were correctly captured by the model.

\section{Positive selection}

To identify key genetic sites affecting the transmissibility of MERS-CoV from animals to human, we performed positive selection analysis on the collected sequences. Based on the phylogenetic tree, we focused on two branches with potentially high selection pressure (Additional file 1: Figure S5a), the branch separating the hedgehog-related clade from the clades among bat, camel and human (branch A), and the one separating the batrelated sequences from clade $\mathrm{C}$ among camel and human (branch B). We tested whether specific sites underwent positive selection along branches A or B using the branch-site test model implemented in the codeML program of the PAML package. For branch A, we identified three proteins (NS3, 1AB polyprotein and nucleoprotein) and 59 sites in the $1 \mathrm{AB}$ polyprotein under positive selection (Additional file 1: Table S8). For branch B, we identified two different proteins (ORF8b and spike glycoprotein) and eight sites in the spike glycoprotein under positive selection. Three of the eight spike glycoprotein sites, 77:Y, 486:H and 636:Q have not been previously reported. No positive selection was detected in the spike glycoprotein for branch A.

\section{Discussion}

Combining updated case data, genetic data and relevant socioenvironmental data, we provided an in-depth analysis of the epidemiology of MERS and genetic evolution of MERS-CoV in the most affected regions. Countries in the Middle East, particularly the Saudi Arabia, continue to be the epicenter of MERS, with frequent animal-to-human spillovers in the region and sporadic exportation of human cases to other continents. Patients $\geq 65$ years old or with underlying conditions had a significantly higher risk of death. The effect of animal contact on the risk of

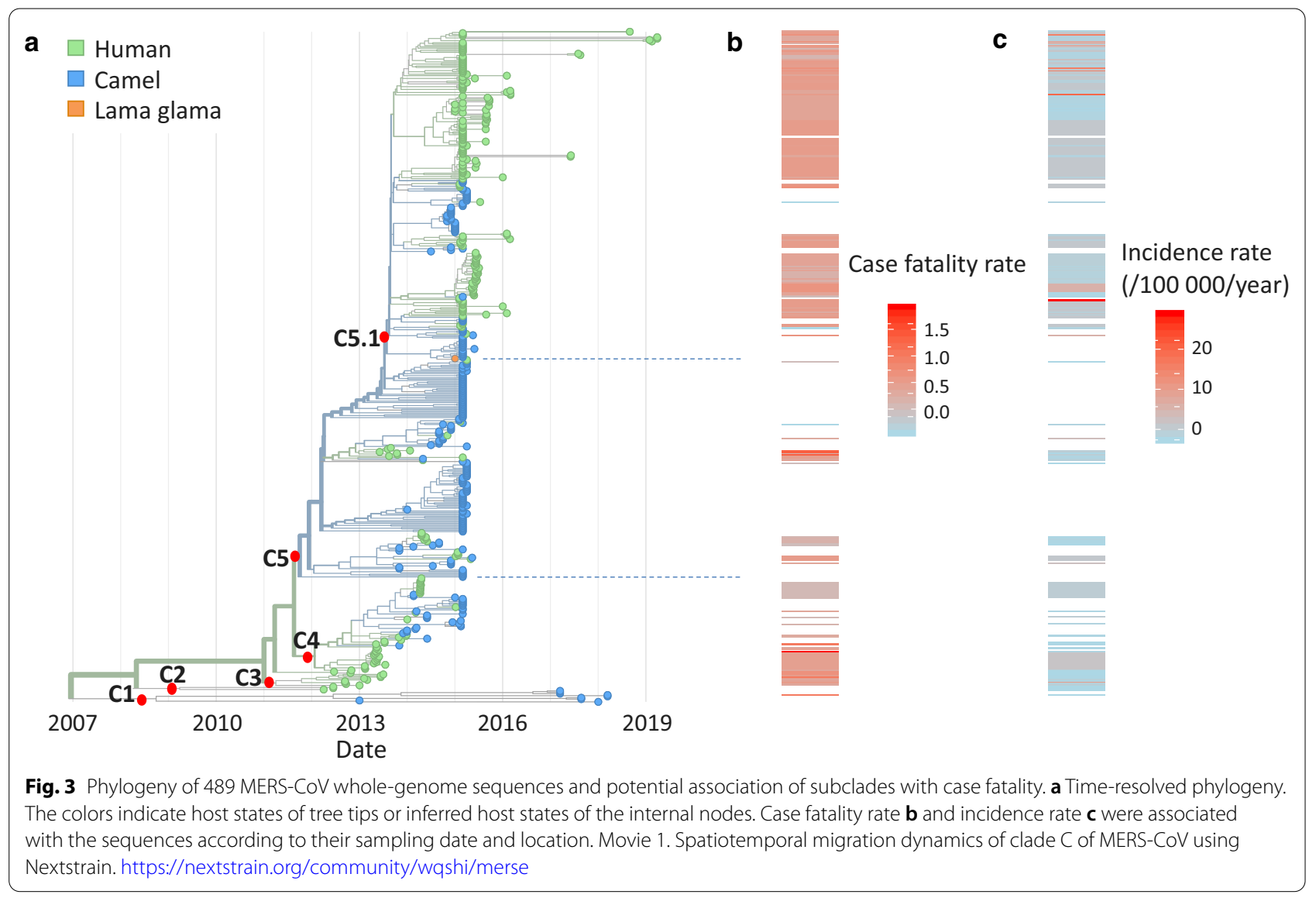


death depends on both age and sex. Transportation network was the leading driver for the spatial diffusion of the disease.

We identified marked differences in demographic and clinical features of cases between the two transmission modes, human-to-human vs animal-to-human, e.g., cases with animal contact tended to be older, more likely to be male and symptomatic, and more likely to have underlying conditions and longer delay of diagnosis. The two modes also differ in seasonality. Animal-to-human transmission events occurred mainly between January and March, and human-to-human transmission peaks subsequently from April to June [24]. This temporal order reflects the importance of blocking animal-to-human spillover in early spring. Preventive measures such as educational campaigns and advocating personal protection equipment among workers of camel farms and trading posts can be used to reduce zoonotic infection in the high-risk season.

Risk factors for case fatality in our analyses includes the elderly ( $\geq 65$ years old), male sex, Middle Eastern residents, underlying diseases, and animal exposure, which are in line with previous findings [31]. Our analyses further revealed that the dependence of the risk of fatality on animal contact history varied by age group and sex. animal contact history was a risk factor for death in female patients $<65$ years old but was protective in male patients $\geq 65$ years old. This observation could be explained by the possibility that, in the Middle East, longterm exposure to dromedary camels has built immunity in older males, and opportunistic exposure of fully susceptible females, especially those younger than 65 years, could be more lethal. This possibility is partly supported by the higher seroprevalence in older males than other age and sex groups [32]. Special attention should be paid and timely treatment should be provided to young female and old male patients upon admission or diagnosis to prevent severe adverse outcomes.

We found the road and railway traffic network played an important role in the rapid regional dispersion of MERS. While the virus has not gained efficient humanto-human transmissibility, the frequent domestic and international migrations of infected humans and animals within the Middle East and between the Middle East and the rest of the world are imposing a sustained risk of viral adaption to human immune system, similar to SARSCoV-2. The outbreak involving 186 cases and 36 deaths in the Republic of Korea highlighted this threat [13]. Surveillance and screening of infected travelers at transportation hubs such as international airports, are needed, especially in areas with frequent travelers from and to the epidemic areas. Avoiding congestion of dromedary herds at watering sites and during their transportation is likely an effective control measure to reduce the circulation of the virus in its natural animal reservoir and thereby to reduce the chance of spillover to humans. Vaccination of dromedary camels could be a better option depending on the development progress and availability of effective vaccines [33].

Our phylogenetic analysis estimated that the inferred root ancestor was $49.3 \%$ likely from camel and 50.7\% likely from human, different from another study that reported camel to be the sole possible host of ancestral root [20]. This gap might result from the differences in model structures and assumptions as well as in sequence samples. In a sensitivity analysis, we down-sampled sequences after 2014 and estimated camel to be the host of root ancestor with a posterior probability of $99.4 \%$, suggesting existing sequences may not represent the true spatiotemporal distribution of the underlying viral population and hence may lead to biased and unstable estimation. Likewise, we also caution the interpretation of the potential association of clade $\mathrm{C} 5$ with a higher mortality rate, which needs to be verified in the future.

Our phylogeographic analysis revealed the dissemination history of MERS-CoV both within the Middle East and from the peninsula to other continents. The spatiotemporal transmission pattern of clade $\mathrm{C}$ was basically consistent with the spatiotemporal distribution of cases at the individual level and early diffusion at the population level. The fact that Abu Dhabi of UAE became the second hub for international exportation of the virus is highly alarming, indicating the necessity of screening MERS-CoV-infected travelers at international airports. However, there still exists inconsistency between some fine details of model-estimated migration trajectories and epidemiological data. For example, the patient who traveled from Saudi Arabia to Chicago was shown by the model as coming from UAE, and the first exportation to East Asia was misplaced at Southern China instead of Republic of Korea [34, 35]. However, such inconsistency in fine details does not alter the big picture of intense local migration in the Middle East and early circulation in the northern and eastern Africa. Future phylogeographic analysis should incorporate prior epidemiological information if such methodology becomes available.

We identified eight amino acid positions in the spike glycoprotein potentially associated with positive selection, three of which are novel sites. The spike glycoprotein is known to mediate viral entry and affect the host range of MERS-CoV and had been previously reported to be under positive selection [36]. The novel sites found in this study may provide research directions for potential targets for development of antivirals and vaccines against MERS-CoV [37]. 
A few limitations of the study are worth mentioning. First, the passive surveillance systems of MERS in most countries only capture patients who sought medical care, while patients with subclinical or asymptomatic infections are usually missed. This surveillance bias may affect our assessment of the relationship between animal contact history and asymptomatic infection. Second, not all relevant risk drivers or confounders were considered in our diffusion analysis and the regression model for case fatality due to lack of data, e.g., the density of dromedary camels and the number of healthcare facilities. Finally, the potential sampling bias in the whole-genome sequences could have affected the phylogenetic, phylogeographic and positive selection analyses, which may not be fully addressable by down-sampling.

\section{Conclusions}

Despite its current incompetence for human-to-human transmission, MERS-CoV has successfully expanded its enzootic range throughout the Middle East, North and East Africa, and West and Southwest Asia, and imposing imminent pandemic threat through genetic mutation or recombination with other human coronaviruses, especially given the widespread of SARS$\mathrm{CoV}-2$. Active surveillance of adapting mutants among human patients and animal reservoir, as well as screening of infected travelers at transportation hubs such as international airports, are urgently needed. While the rapid development of SARS-CoV-2 vaccines shed lights on how to advance MERS-CoV vaccine candidates, nonpharmaceutical interventions and animal vaccines should be planned ahead to delay or block the adaption of MERS-CoV at the source.

\section{Abbreviations}

CDS: Coding sequences; CFR: Case fatality rate; Cl: Confidence Interval; FAO: Food and Agriculture Organizationon; HR: Hazard ratio; IQR: Interquartile range; MERS: Middle East respiratory syndrome; MERS-CoV: Middle East respiratory syndrome coronavirus; OR: Odds ratio; ORF: Open reading frame; OTC: Time from disease onset to confirmation; WHO: World Health Organization.

\section{Supplementary Information}

The online version contains supplementary material available at https://doi. org/10.1186/s40249-021-00853-0.

Additional file 1: Additional tables and figures.

\section{Acknowledgements}

Not applicable.

\section{Authors' contributions}

LQF, WL, YY and WM conceived and designed the study. ARZ, KL, and WQS performed the main data collection and analyses under supervision of LQF,
WL, YY and WM. XLL, MJL, WHZ, GPZ, JJC, XAZ, and DM helped with the analyses. WL, ARZ, KL, WQS, LQF and $Y Y$ wrote the draft of the manuscript. All authors contributed to and approved the final version of the manuscript. All authors read and approved the final manuscript.

\section{Funding}

This work was supported by China Mega-Project on Infectious Disease Prevention (No. 2017ZX10303401, 2018ZX10713002, 2018ZX10101003 and 2018ZX10201001), National Natural Science Foundation of China (No. 81825019), and the National Institutes of Health of United States (R01 Al139761 and R01 Al116770). YY was supported by US National Institutes of Health grants R01 Al139761 and R56 Al148284.

Availability of data and materials

Please contact author for data requests.

\section{Declarations}

\section{Ethics approval and consent to participate}

This research was considered exempt from ethical review because it is limited to secondary data analysis based on publicly available data, which contains no information that can directly or indirectly identify an individual.

\section{Consent for publication}

Not applicable.

\section{Competing interests}

All authors declare no competing interests.

\section{Author details}

${ }^{1}$ Department of Epidemiology, School of Public Health, Cheeloo College of Medicine, Shandong University, 44 West Wenhua Road, Jinan, People's Republic of China. ${ }^{2}$ State Key Laboratory of Pathogen and Biosecurity, Beijing Institute of Microbiology and Epidemiology, 20 Dong-Da Street, Fengtai District, Beijing 100071, People's Republic of China. ${ }^{3}$ Department of Biostatistics, College of Public Health and Health Professions, and Emerging Pathogens Institute, University of Florida, Gainesville, FL, USA. ${ }^{4}$ Department of Epidemiology, Ministry of Education Key Lab of Hazard Assessment and Control in Special Operational Environment, School of Public Health, Air Force Medical University, Xi'an, People's Republic of China. ${ }^{5}$ Department of Medical Research, Key Laboratory of Environmental Sense Organ Stress and Health of the Ministry of Environmental Protection, PLA Stragetic Support Force Characteristic Medical Center, Beijing, People's Republic of China. ${ }^{6}$ Logistics College of Chinese People's Armed Police Forces, Tianjin, People's Republic of China.

Received: 3 March 2021 Accepted: 27 April 2021

Published online: 08 May 2021

\section{References}

1. Zaki AM, van Boheemen S, Bestebroer TM, Osterhaus ADME, Fouchier RAM. Isolation of a novel coronavirus from a man with pneumonia in Saudi Arabia. N Engl J Med. 2012;367:1814-20. https://doi.org/10.1056/ NEJMoa1211721.

2. World Health Organization. Middle East respiratory syndrome coronavirus (MERS-CoV). http://www.who.int/emergencies/mers-cov/en/. Accessed 30 Jan 2020.

3. Memish ZA, Zumla A, Alhakeem RF, Assiri A, Turkestani A, Al Harby $K D$, et al. Hajj: infectious disease surveillance and control. Lancet. 2014;383:2073-82. https://doi.org/10.1016/S0140-6736(14)60381-0.

4. Mehand MS, Al-Shorbaji F, Millett P, Murgue B. The WHO R\&D Blueprint: 2018 review of emerging infectious diseases requiring urgent research and development efforts. Antiviral Res. 2018;159:63-7. https://doi.org/10. 1016/j.antiviral.2018.09.009.

5. David D, Rotenberg D, Khinich E, Erster O, Bardenstein S, van Straten M, et al. Middle East respiratory syndrome coronavirus specific antibodies in naturally exposed Israeli llamas, alpacas and camels. One Health. 2018;5:65-8. https://doi.org/10.1016/j.onehlt.2018.05.002. 
6. Anthony SJ, Gilardi K, Menachery VD, Goldstein T, Ssebide B, Mbabazi R, et al. Further evidence for bats as the evolutionary source of Middle East respiratory syndrome coronavirus. MBio. 2017;8:e00373-e417. https://doi. org/10.1128/mBio.00373-17.

7. Chan RW, Hemida MG, Kayali G, Chu DK, Poon LL, Alnaeem A, et al. Tropism and replication of Middle East respiratory syndrome coronavirus from dromedary camels in the human respiratory tract: an in-vitro and ex-vivo study. Lancet Respir Med. 2014;2:813-22. https://doi.org/10.1016/ S2213-2600(14)70158-4.

8. Killerby ME, Biggs HM, Midgley CM, Gerber SI, Watson JT. Middle East respiratory syndrome coronavirus transmission. Emerg Infect Dis. 2020;26:191-8. https://doi.org/10.3201/eid2602.190697.

9. Cotten M, Watson SJ, Kellam P, Al-Rabeeah AA, Makhdoom HQ, Assiri A, et al. Transmission and evolution of the Middle East respiratory syndrome coronavirus in Saudi Arabia: a descriptive genomic study. Lancet. 2013;382:1993-2002. https://doi.org/10.1016/S0140-6736(13)61887-5.

10. Lau SKP, Wong ACP, Lau TCK, Woo PCY. Molecular evolution of MERS coronavirus: Dromedaries as a recent intermediate host or long-time animal reservoir? Int J Mol Sci. 2017;18:2138. https://doi.org/10.3390/ijms181021 38.

11. Nam HS, Park JW, Ki M, Yeon MY, Kim J, Kim SW. High fatality rates and associated factors in two hospital outbreaks of MERS in Daejeon, the Republic of Korea. Int J Infect Dis. 2017;58:37-42. https://doi.org/10. 1016/j.ijid.2017.02.008.

12. Memish ZA, Zumla Al, Assiri A. Middle East respiratory syndrome coronavirus infections in health care workers. N Engl J Med. 2013;369:884-6. https://doi.org/10.1056/NEJMc1308698.

13. Xia ZQ, Zhang J, Xue YK, Sun GQ, Jin Z. Modeling the transmission of Middle East respirator syndrome coronavirus in the Republic of Korea. PLoS One. 2015;10:e0144778. https://doi.org/10.1371/journal.pone.0144778.

14. Alenazi TH, Al Arbash $\mathrm{H}$, El-Saed A, Alshamrani MM, Baffoe-Bonnie $H_{\text {, }}$ Arabi YM, et al. Identifed transmission dynamics of Middle East respiratory syndrome coronavirus infection during an outbreak: implications of an overcrowded emergency department. Clin Infect Dis. 2017;65:675-9. https://doi.org/10.1093/cid/cix352.

15. Hui DS, Azhar El, Kim YJ, Memish ZA, Oh MD, Zumla A. Middle East respiratory syndrome coronavirus: risk factors and determinants of primary, household, and nosocomial transmission. Lancet Infect Dis. 2018;18:e217-27. https://doi.org/10.1016/S1473-3099(18)30127-0.

16. Alraddadi BM, Watson JT, Almarashi A, Abedi GR, Turkistani A, Sadran M, et al. Risk factors for primary Middle East respiratory syndrome coronavirus illness in humans, Saudi Arabia, 2014. Emerg Infect Dis. 2016;22:49-55. https://doi.org/10.3201/eid2201.151340.

17. Banik GR, Alqahtani AS, Booy R, Rashid H. Risk factors for severity and mortality in patients with MERS-CoV: Analysis of publicly available data from Saudi Arabia. Virol Sin. 2016;31:81-4. https://doi.org/10.1007/ s12250-015-3679-z.

18. Sajini AA, Alkayyal AA, Mubaraki FA. The recombination potential between SARS-CoV-2 and MERS-CoV from cross-species spill-over infections. J Epidemiol Glob Health. 2020. https://doi.org/10.2991/jegh.k. 201105.001.10.2991/jegh.k.201105.001.

19. Kim JI, Kim YJ, Lemey P, Lee I, Park S, Bae JY, et al. The recent ancestry of Middle East respiratory syndrome coronavirus in Korea has been shaped by recombination. Sci Rep. 2016;6:1-7. https://doi.org/10.1038/srep1 8825.

20. Dudas G, Carvalho LM, Rambaut A, Bedford T. MERS-CoV spillover at the camel-human interface. Elife. 2018;7:e31257. https://doi.org/10.7554/ elife.31257.

21. Chen X, Adam DC, Chughtai AA, Stelzer-Braid S, Scotch M, Maclntyre CR. The phylogeography of MERS-CoV in hospital outbreak-associated cases compared to sporadic cases in Saudi Arabia. Viruses. 2020;12(5):540. https://doi.org/10.3390/v12050540.

22. Hadfield J, Megill C, Bell SM, Huddleston J, Potter B, Callender C, et al. Nextstrain: real-time tracking of pathogen evolution. Bioinformatics. 2018;34:4121-3. https://doi.org/10.1093/bioinformatics/bty407.

23. Katoh K, Standley DM. MAFFT multiple sequence alignment software version 7: improvements in performance and usability. Mol Biol Evol. 2013;30:772-80. https://doi.org/10.1093/molbev/mst010.

24. Nguyen LT, Schmidt HA, von Haeseler A, Minh BQ. IQ-TREE: a fast and effective stochastic algorithm for estimating maximum-likelihood phylogenies. Mol Biol Evol. 2015;32:268-74. https://doi.org/10.1093/molbev/ msu300.

25. Sagulenko P, Puller V, Neher RA. TreeTime: maximum-likelihood phylodynamic analysis. Virus Evol. 2018. https://doi.org/10.1093/ve/vex042.

26. Suyama M, Torrents D, Bork P. PAL2NAL: robust conversion of protein sequence alignments into the corresponding codon alignments. Nucleic Acids Res. 2006;34:W609-12. https://doi.org/10.1093/nar/gkl315.

27. Yang Z. PAML 4: phylogenetic analysis by maximum likelihood. Mol Biol Evol. 2007;24:1586-91. https://doi.org/10.1093/molbev/msm088.

28. Huerta-Cepas J, Serra F, Bork P. ETE 3: Reconstruction, analysis, and visualization of phylogenomic data. Mol Biol Evol. 2016;33:1635-8. https://doi. org/10.1093/molbev/msw046.

29. Chu DK, Poon LL, Gomaa MM, Shehata MM, Perera RA, Abu Zeid D, et al. MERS coronaviruses in dromedary camels. Egypt Emerg Infect Dis. 2014;20:1049-53. https://doi.org/10.3201/eid2006.140299.

30. Poletto C, Colizza V, Boëlle PY. Quantifying spatiotemporal heterogeneity of MERS-CoV transmissionin the Middle East region: A combined modelling approach. Epidemics. 2016;15:1-9. https://doi.org/10.1016/j.epidem. 2015.12.001.

31. Rivers CM, Majumder MS, Lofgren ET. Risks of death and severe disease in patients with Middle East respiratory syndrome coronavirus, 2012-2015. Am J Epidemiol. 2016;184:460-4. https://doi.org/10.1093/aje/kww013.

32. Degnah AA, Al-Amri SS, Hassan AM, Almasoud AS, Mousa M, Almahboub SA, et al. Seroprevalence of MERS-CoV in healthy adults in western Saudi Arabia, 2011-2016. J Infect Public Health. 2020;13:697-703. https://doi. org/10.1016/j.jiph.2020.01.001.

33. Widagdo W, Okba NMA, Stalin Raj V, Haagmans BL. MERS-coronavirus: from discovery to intervention. One Health. 2016;3:11-6. https://doi.org/ 10.1016/j.onehlt.2016.12.001.

34. Bialek SR, Allen D, Alvarado-Ramy F, Arthur R, Balajee A, Bell D, et al. First confirmed cases of Middle East respiratory syndrome coronavirus (MERS$\mathrm{CoV}$ ) infection in the United States, updated information on the epidemiology of MERS-CoV infection, and guidance for the public, clinicians, and public health authorities-May 2014. MMWR Morb Mortal Wkly Rep. 2015;63:431-6.

35. Wu J, Yi L, Zou L, Zhong H, Liang L, Song T, et al. Imported case of MERS-CoV infection identified in China, May 2015: detection and lesson learned. Euro Surveill. 2015;20:21158. https://doi.org/10.2807/1560-7917. es2015.20.24.21158.

36. Zhang Z, Shen L, Gu X. Evolutionary dynamics of MERS-CoV: potential recombination, positive selection and transmission. Sci Rep. 2016;6:25049. https://doi.org/10.1038/srep25049.

37. Qian Z, Dominguez SR, Holmes KV. Role of the spike glycoprotein of human Middle East respiratory syndrome coronavirus (MERS-CoV) in virus entry and syncytia formation. PLoS One. 2013;8:e76469. https://doi. org/10.1371/journal.pone.0076469. 